\title{
Vaginal Blue Nevus
}

National Cancer Institute

\section{Source}

National Cancer Institute. Vaginal Blue Nevus. NCI Thesaurus. Code C40282.

A neoplasm that arises from the vagina and is characterized by the proliferation of subepithelial benign dendritic melanocytes. 